\title{
Neurocognitive Empowerment for Addiction Treatment (NEAT): Study Protocol for a Randomized Controlled Trial
}

\section{Authors:}

1. Hamed Ekhtiari ${ }^{*}$, Laureate Institute for Brain Research, Tulsa, OK, USA. hekhtiari@laureateinstitute.org

2. Tara Rezapour, Institute for Cognitive Science Studies, Tehran, Iran. rezapour_t@iricss.org

3. Brionne Sawyer, Laureate Institute for Brain Research, Tulsa, OK, USA. bsawyer@laureateinstitute.org

4. Hung-Wen Yeh, Children's Mercy Hospital, Kansas City, Mo, USA.

hyeh@cmh.edu

5. Rayus Kuplicki, Laureate Institute for Brain Research, Tulsa, OK, USA. rkuplicki@laureateinstitute.org

6. Mimi Tarrasch, Family and Children Services, Tulsa, OK, USA.

mtarrasch@fcsok.org

7. Martin Paulus, Laureate Institute for Brain Research, Tulsa, OK, USA. mpaulus@laureateinstitute.org

8. Robin Aupperle, Laureate Institute for Brain Research, Tulsa, OK, USA. raupperle@laureateinstitute.org

Corresponding Author:

Hamed Ekhtiari, MD, PhD

Laureate Institute for Brain Research, 6655 South Yale Ave. Tulsa, OK 74136

Email: hekhtiari@laureateinstitute.org 


\begin{abstract}
Background: Neurocognitive deficits (NCDs) and associated meta-cognition difficulties associated with chronic substance use often delay the learning and change process necessary for addiction recovery and relapse prevention. However, very few cognitive remediation programs have been developed to target NCDs and meta-cognition for substance users. The study described herein aims to investigate the efficacy of a multi-component neurocognitive rehabilitation and awareness program termed 'Neurocognitive Empowerment for Addiction Treatment'(NEAT). NEAT is a fully manualized, cartoon-based intervention involving psychoeducation, cognitive practice, and compensatory strategies relevant across 10 major cognitive domains, including aspects of attention, memory, executive functions, and decision-making.

Method/design: In a single-blind randomized controlled trial (RCT), 80 female opioid and/or methamphetamine users will be recruited from an addiction recovery program providing an alternative to incarceration for women with substance use related offenses. Eight groups of 9-12 participants will be randomized into NEAT or treatment-as-usual (TAU). NEAT involves 14 90-min sessions, delivered twice weekly. The primary outcome is change in self-reported drug craving from before to after intervention using obsessive compulsive drug use scale. Secondary and exploratory outcomes include additional psychological, neurocognitive and structural and functional neuroimaging measures. Clinical measures will be performed at five time points (pre- and post- intervention, 3-, 6-, and 12-month follow-up); neuroimaging measures will be completed at pre- and post-intervention.

Discussion: The present RCT is the first study to examine the efficacy of an adjunctive neurocognitive rehabilitation and awareness program for addiction. Results from this study will provide initial information concerning potential clinical efficacy of the treatment, as well as delineate neural mechanisms potentially targeted by this novel intervention.

Trial registration: This trial was registered in clinicaltrials.gov on 22 April 2019 with NCT03922646 as clinicalTrials.gov identifier.
\end{abstract}

Keywords: opioid, methamphetamine, neurocognitive deficits, meta-cognition, rehabilitation, awareness, addiction, substance use disorder, diversion program, women.

Word number: 7660

\title{
1. Background
}

Opioid use disorder (OUD) and methamphetamine use disorder (MUD) are among the costliest mental health disorders in the United States and worldwide ${ }^{1,2}$. Opioids (including prescription pain relievers and the illicit drug heroin) were involved in more than 42,000 deaths in the United States in 2016, more than any year on record ${ }^{3}$. This accounts for more deaths than road accidents and gun violence combined. In 2017, amphetamine use disorders were reported as the second most common use disorders world-wide by the United Nations Office on Drugs and Crimes (UNODC) ${ }^{4}$. Even more alarming, the number of overdoses with heroin and methamphetamine has tripled from 2011 to $2016^{5}$.

Chronic OUD and MUD are associated with numerous other mental health symptoms, including anxiety and depression ${ }^{6,7}$, as well as subjective and objective neural and cognitive deficits. These neurocognitive deficits (NCDs) are apparent across domains of memory, attention, decision-making, judgment, negative affect, metacognition, self-regulation and impulse inhibition ${ }^{8-10}$. Neuroimaging studies indicate that these deficits may relate to dysfunction within a broad network of brain regions, including prefrontal cortex, orbitofrontal cortex, amygdala, nucleus accumbens, hippocampus, and anterior cingulate gyrus ${ }^{11-13}$. Maladaptive neural changes in these regions results in aberrant salience attribution, increased reward expectancy, changed learning pattern (including conditioned-incentive learning, habit learning, declarative learning), and impulsive decision making ${ }^{13}$. 
Current treatment programs for OUDs and MUDs are mainly focused on abstinence from illicit drugs, with the assumption that the NCDs will be subsequently restored. However, NCDs are found to persist or sometimes even become exacerbated after long-term abstinence and are thought to contribute to relapse, decreased quality of life, and/or lack of reintegration into society ${ }^{14}$. Furthermore, NCDs (particularly those related to attention and memory) are considered a potential predictor for treatment outcomes for either the core substance use disorder or co-occurring symptoms (i.e., cognitive-behavioral therapies) ${ }^{15,16}$.

Numerous cognitive rehabilitation programs have been developed to focus on compensatory strategies and restorative exercises for NCDs associated with traumatic brain injuries ${ }^{17}$, stroke ${ }^{18}$, multiple sclerosis ${ }^{19}$ and schizophrenia ${ }^{20}$. These programs have consistently been found to improve functioning and long-term outcomes for these groups of patients. There have been a few preliminary attempts to transplant cognitive rehabilitation programs for use with substance use populations, providing initial evidence that such programs could be beneficial. However, these programs did not explicitly link the cognitive rehabilitation modules to the processes involved in addiction and recovery. There is potential value in clarifying how such strategies may relate to the current neuroscientific understanding of addiction and recovery, as well as the co-occurring symptoms often experienced.

The present pilot study aims to characterize the clinical potential for an intervention targeting NCDs in OUDs and MUDs by enhancing awareness and use of neurocognitive skills in the context of substance use recovery. This aim will be accomplished by randomizing subjects who are already enrolled in substance use treatment to complete a novel "Neurocognitive Empowerment for Addiction Treatment" (NEAT) program or treatment-as-usual intervention. NEAT is novel in (a) its use of cartoons, brain awareness games and real-life scenarios to ensure it is interactive and engaging, (b) its focus on the role of neurocognitive deficits in recovery from substance use and co-occurring mental health symptomatology, and (c) its incorporation of neuroscience findings specific to substance use.

We hypothesis that as compared to the active control, the NEAT program will be associated with:

1. greater reduction in self-report drug carving symptom from before to after intervention (primary outcome).

2. enhanced subjective cognitive functioning from before to after intervention (secondary outcome).

3. improved objective neuropsychological functioning from before to after intervention.

4. reduced mental health (i.e., depression and anxiety) symptoms from before to after intervention and other follow up time-points.

5. lower number of relapses as measured with urine drug tests during follow up time points.

6. decreases in subcortical limbic (amygdala and ventral striatum) activation and increase prefrontal cortical top-down inhibitory activation during induced drug craving in fMRI drug cue reactivity from before to after intervention.

7. increases in cortical gray matter density (measured with voxel-wise brain morphometry) and white matter track integrity (measured with fractional anisotropy) in areas related to self-control and awareness, including prefrontal gray matter and frontostriatal/frontoamygdalar white matter from before to after intervention.

Outcome measures 3. to 7. are exploratory.

\section{Methods/Design}

\subsection{Study objectives:}

The present study aims to (a) determine the potential clinical impact of the NEAT program on clinical symptoms and function, from pre- to post- intervention and at long-term follow up (12 months after treatment completion); and (b) explore potential impact of the NEAT program on functional and structural brain recovery, from pre- to post- intervention. 


\subsection{Research environment/context}

The present trial is conducted by Laureate Institute for Brain Research and participants are recruited from a single site, the Women in Recovery (WIR) program at Family and Children's Services located in Tulsa, Oklahoma. WIR is an intensive outpatient alternative for eligible women facing long prison sentences for non-violent drug-related offenses.

\subsection{Design}

This is an assessor-blinded, two-arm, parallel group, randomized controlled clinical trial formulated in accordance with the Consolidated Standards of Reporting Trials (CONSORT) 2010 guidelines (supplementary file 1). This protocol was written using the Standard Protocol Items: Recommendations for Interventional Trials (SPIRIT) guidelines, and the SPIRIT checklist is provided in supplementary file 2. 80 participants will be recruited in eight groups. Groups will be randomly allocated to intervention $(n=40)$ and control $(n=40)$ groups in blocks of 4 in a 1:1 ratio. All 80 participants will receive treatment as usual (TAU). Those subjects allocated to the intervention group will receive TAU but will complete NEAT in lieu of nonessential aspects of the TAU program that do not explicitly target neurocognitive function (e.g., culinary or legal education classes). Thus, both NEAT and TAU groups received similar amount (e.g., intensity, duration, frequency) of intervention. Self-report, interview-based, and neuroimaging assessments will be performed before (pre; week=0), during intervention (from week=1 to week=7), after the intervention period (post; week=8) and/or after a follow-up period $(3-, 6-, 12$ - month; week=20,32,56, respectively). Interview-based assessments were conducted by a trained clinical practitioner blind to the allocation of participants. Figure 1 summarizes the full design of the trial in accordance with CONSORT flow diagram.

The trial was approved by Western Institutional Review Board (approval number: 20181028, 1-11414031) and registered in clinicaltrials.gov on 22 April 2019 with NCT03922646 as clinicalTrials.gov identifier. No amendments have been made to the protocol since original submission to ClinicalTrials.gov. The study is funded by the Oklahoma Center for Advancement of Science and Technologies (OCAST) Hamed Ekhtiari $[\mathrm{HE}]$, principal investigator $[\mathrm{PI}]$ and Robin Aupperle [RA] as co-investigator $[\mathrm{CI}])$.

\subsection{Participants}

\subsubsection{Inclusion criteria}

To participate in this trial, subjects must (1) be 18 and 55 years old; (2) enrolled in WIR within 6 months, (3) diagnosed with OUD and/or MUD (based on the Mini-International Neuropsychiatric Interview (MINI) diagnostic interview for DSM- $5^{21}$ ) over the last 12 months; (4) be able to provide written informed consent; (5) have sufficient proficiency in the English language to understand and complete interviews, questionnaires, and all other study procedures; and (6) have completed at least an 8th grade education, to help facilitate ability to engage in the written materials included in the NEAT program.

\subsubsection{Exclusion criteria}

Potential subjects will be excluded if they (1) are reluctant or unable to complete any of the major aspects of the study protocol, including self-report or behavioral assessment; (2) have uncorrectable vision or hearing problems that would interfere with completion of study procedures; (3) have diagnoses of Schizophrenia spectrum or other psychotic disorders; (4) have current mental or physical health symptoms that require immediate attention (such as suicidal ideation with intent or plan; active psychotic symptoms; delirium; etc.); (5) have a history of unstable liver or renal insufficiency; glaucoma; significant and unstable cardiac, vascular, pulmonary, gastrointestinal, endocrine, neurologic, hematologic, rheumatologic, or metabolic disturbance; or any other condition that, in the opinion of the investigator, would make participation not be in the best interest (e.g., compromise the well-being) of the subject or 
that could prevent, limit, or confound the protocol-specified assessments; (6) have a positive test for drugs of abuse at the time of baseline assessment, including alcohol, cocaine, marijuana, opiates, amphetamines, methamphetamines, phencyclidine, benzodiazepines, barbiturates, methadone, and oxycodone; (7) have MRI contraindications; and (8) have moderate to severe traumatic brain injury (loss of consciousness for less than $30 \mathrm{~min}$ or $>24$ hours posttraumatic amnesia) or other neurocognitive disorder with evidence of neurological deficits, neurological disorders, or severe or unstable medical conditions that might be compromised by participation in the study.

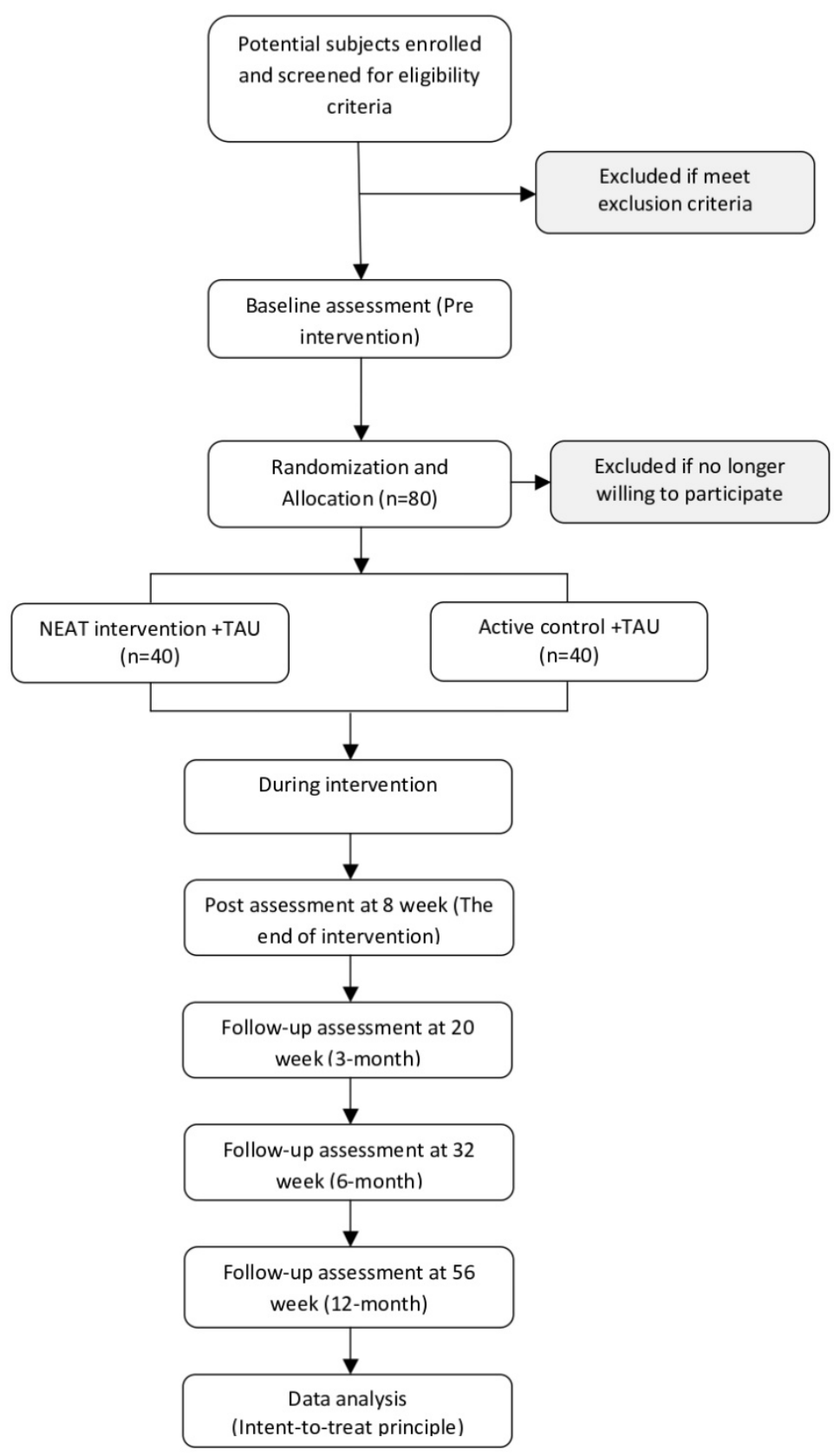

Fig. 1 CONSORT flow diagram. The flow chart depicts participant progression through the study from initial enrolment through allocation, follow-up and finally analyses of their data stages. Planned sample size, $n=64$; to be enrolled, $n=80$. Having 40 people in each arm will be subject to drop out. 


\subsection{Sample size calculation}

With total enrollment of $\mathrm{N}=80$ and an estimated $20 \%$ attrition rate, we anticipate having complete data at our primary endpoint (post-treatment) for 32 subjects per group or 64 in total. Using a continuous measure (self-reported drug craving) with a precision of $t_{n-1,1-\alpha / 2} / \sqrt{n}=2.04 / \sqrt{32}=0.36$ times standard deviation as margin of error at $95 \%$ confidence, we estimate having $80 \%$ power to detect medium to large effect sizes $(d=0.71)$ for group differences in pre to post or pre to follow up changes at a two-sided $5 \%$ significance of a 2-sample t-test. While we may be underpowered to detect small to medium effects, the current pilot study will be crucial for informing the viability of the intervention and for estimating power for future, larger clinical trials.

\subsection{Recruitment}

Study participants will be recruited through WIR program at Family and Children's Services. Flyers will be provided to clinic program staff to be given to potential subjects. The research team may also discuss the study to groups of patients (i.e., when gathered for a class). Research team will be available at WIR on a regular basis to discuss the study privately with subjects interested in the study. WIR staff will not be present during these individual meetings in order to increase confidentiality and ensure that subjects are making an informed decision to participate. All efforts will be made to ensure that the recruited subject population closely resembles the ethnic and racial composition of the WIR program. The enrolled participants then will give a written informed consent to one of the principle investigators (PI) or research coordinator (RC) in a private interview/exam room at LIBR or WIR. Family members will be allowed to be present and discuss the consenting process with the participant if requested. In this session, it will be emphasized that participation is strictly voluntary and that they have the right to withdraw at any time without penalty. It will be emphasized that the information provided during the course of the study will have no impact on participants' treatment and care provided by WIR or other programs and clinics, their experience at correctional facilities, or on any court processes or decisions.

Noted that participants will be monetarily compensated for their time of participation in self-report, behavioral and neuroimaging assessment. Each participant will be also provided with a gift card (\$10-15 per session) to compensate completing intervention session assessments.

\subsection{Randomization and Blinding}

All consented participants will receive NEAT or other active, but non-essential regularly scheduled programming at WIR coinciding with the same timeframe. Recruited subjects will be randomly allocated to intervention conditions (study arms) in groups of 9-12 participants. This randomization will be conducted in blocks of 4 groups. The randomization will be determined prior to enrolling any subjects using computer generated allocations.

The randomization codes will be generated by a statistician not involved in enrollment or delivery of interventions. Research staff involved in the conduct of interview-based assessments (e.g., MINI, PRISM5) will be blinded to intervention condition. Participants and all research staff are kept blind to their intervention condition until completion of all baseline assessments. However, due to the nature of the intervention, participants and research staff involved in providing interventions will not be kept blind to interventions after completion of baseline assessments.

\subsection{Treatment as usual}

All participants will complete the essential components of the WIR treatment program. The WIR program includes daily and weekly clinical visits and both individual and group sessions. These sessions include but are not limited to cognitive behavioral therapy, cognitive processing therapy, motivational interviewing, behavioral modification and strength-based case management interventions, nutritional education, and occupational training. The WIR program is conducted in three phases, with increasing independence at 
each phase (e.g., supervised probation with ankle monitor and residing in program-supervised housing at first; independent housing, independent employment, and outpatient visits multiple times per week towards the end of the program). Phase one includes 40 structured hours weekly that is devoted to addiction and trauma treatment, parent education, job readiness training, life skills, safe group housing, education, and community volunteering. Phase two includes 24 structured hours weekly that is devoted to job retention training, part-time job replacement, parent-child visitation, health and wellness services, education groups, subsidized housing, financial literacy, and recovery support. Phase three includes 12 structured hours weekly that in considered full time employment, parent-child reunification, education groups, independent housing, recovery support technology, and community integration. Graduation from the program occurs after completion of phase 3 , an average of 17 months in the program. Random drug testing is conducted by WIR throughout participation in the program. After graduation, women can continue participating in group programming on a volunteer basis.

\subsection{Interventions}

\subsubsection{Active control intervention}

Participants in the comparison group will receive the essential components of the WIR program plus WIR training sessions related to health and wellness, communication skills and/or financial literacy, scheduled at the same days/times as the NEAT sessions. Thus, the comparison intervention has the same duration and frequency as the active intervention.

\subsubsection{Active intervention}

The active intervention in this trial, the Neurocognitive Empowerment for Addiction Treatment (NEAT), was developed to target cognitive domains thoughts to be either influenced by substance use, or involved in drug craving and seeking behavior based on the dynamic model of craving (DCM) ${ }^{22}$. According to the DCM model (Figure 2) which has been inspired by the traditional cognitive behavioral therapy frameworks ${ }^{23}$, drug craving is triggered by environmental cues (E) (including both internal and external cues). These cues then elicit distinct yet collaborative bottom-up and top-down attentional processes that increase the focus of attention on drug-relevant cues (A). This attentional deployment activates saliency evaluation processes $(\mathrm{S})$ related to drug-associated cues. The retrieval of memories $(\mathrm{M})$ linked to the drug cues provides relevant information during saliency processing. Saliency evaluation can result in an interoceptive state that represents the subjective feeling of craving, leading to the engagement of executive control (Co) and decision-making processes to execute actions supporting drug-taking behavior or abstinence. In targeting these functions, NEAT also provides skills important for daily functioning and re-integration into society, including self-monitoring, time-management and problem solving ${ }^{24}$.

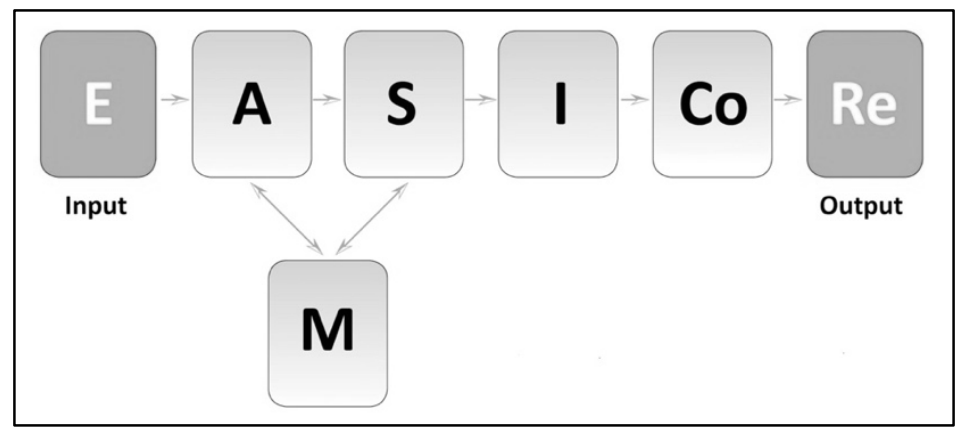

Figure 2. Dynamic model of craving (DCM). A neuroscience informed conceptual framework to define the major cognitive targets in the dynamic response to drug-related or emotionally salient cues that can end up to drug seeking and consumption behaviors. $E=$ Environment; $A=$ Attention, $S=S a l i e n c y$ processing; $M=$ Memory; $I=$ Interoception; $\mathrm{CO}=$ Control; $\mathrm{R}=$ Response 
NEAT consists of 14, 90-min group sessions, which will be led by two trained master's level therapists or therapists-in-training (e.g., doctoral students in clinical psychology) supervised by a licensed clinical psychologist (RLA). Groups are led by therapists according to session-by-session manual. All sessions will be audio-recorded to support supervision and fidelity of implementation. Cartoon-based treatment binders and brain planners are provided to all participants.

Each session starts with a didactic, psychoeducation portion describing the concepts and skills of focus in each session. The session is followed by written material with descriptions of the concepts/skills in verbal and pictorial (cartoon) formats. The session continues with practicing cognitive tasks/games relevant for each skill and ends with discussion of how they can monitor and practice each skill in their everyday life. Beside the in-session components, subjects are provided with homework material to practice the learned objectives between sessions, including monitoring their use of various brain functions, implementing compensatory strategies, and completing additional cognitive exercises. They are also asked to complete aspects of the brain planner between each session, starting with retrospective recording of events and slowly progressing towards prospective planning. NEAT will target the cognitive domains shown in Figure 3 through the use of the following five components:

1. Neuroscience-informed Psychoeducation: The main purpose of the neuroscience-informed psychoeducation component is to promote subjects' awareness about the cognitive impairments often associated with drug craving and addiction. Participants are provided with information concerning common behavioral manifestation of these impairments in daily life activities. They are informed about lifestyle changes (e.g., nutrition, physical exercise, sleep) that can facilitate brain recovery. To improve learning and consolidation, the education components are accompanied by colorful and comic cartoons ${ }^{25}$.

2. Cognitive exercises: According to previous work, using cognitive exercises with game-like features can be useful for supporting treatment engagement and restoration of cognitive functions among people with SUDs ${ }^{26}$. These game-based exercises include such things as Stroop-like tasks ${ }^{27}$,Spot the differences game ${ }^{28}$, target cancellation tasks ${ }^{29}$, and so on. In NEAT, these games are used not only as a way of practicing and improving specific brain functions, but also for enhancing understanding of the brain functions discussed (e.g., as way of demonstrating what is meant by "inhibition" or "working memory") and supporting metacognitive awareness (the next component).

3. Metacognitive training: Metacognition awareness ${ }^{30,31}$ is supported through self-monitoring in between sessions and group discussions about the cognitive functions involved in each brain exercise and the role that these functions (and the associated compensatory strategies) play in their day-to-day functioning, craving management and substance use recovery. This component targets insight towards the cognitive foundations of the training program and aims to enhance motivation to implement skills and generalization of learning.

4. Compensatory strategies: The main purpose of these components is to provide specific cognitive strategies for supporting optimal cognitive functioning and compensating for deficits. For example, providing mnemonic aids and goal management training for memory- and planningrelated problems ${ }^{32,33}$. These components are integrated with the cognitive exercises discussed above to provide a thorough understanding and awareness for the role of specific cognitive functions in their lives.

5. Brain planner: Training participants to keep records of their daily activities, including both retrospectively (e.g., recording past events) and prospectively (e.g., planning future activities and goals), is another critical aspect of NEAT. Planner use can support optimal daily functioning and the use of higher-order cognitive functions such as self-monitoring and planning (which are also supported by compensatory strategies discussed above) but can also help compensate for deficits 
in these cognitive domains and others (i.e., memory deficits) ${ }^{34}$. The use of a planner also supports compliance with NEAT and other substance use treatment activities. Each training session of NEAT has time dedicated to learning and practicing using the planner.

\subsection{Measures}

Table 1 indicates the list of self-report, behavioral, and neuroimaging assessments included in the protocol (refer to Fig. 4 for timing of assessments). We will use the total score of Obsessive Compulsive Drug Use Scale $(O C D U S)^{35}$ as a primary outcome measure for drug craving in the past week. It is a 12 -item questionnaire consisting of three factors including: thoughts about drugs and interference, desire and control, and resistance against thoughts and intention to use drugs that compose a total score.

The remaining tasks used as secondary and exploratory outcome measures are described in below.

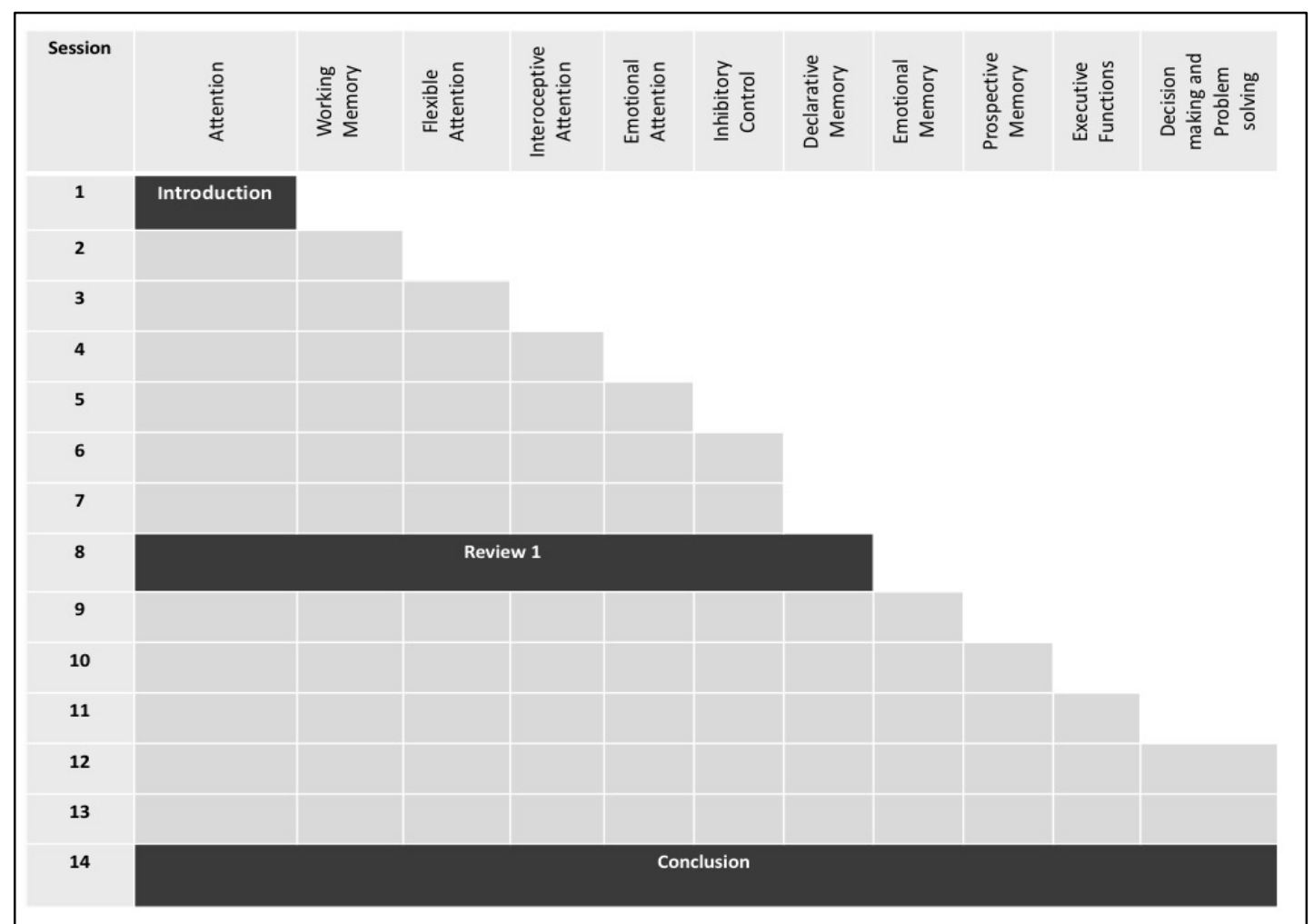

Figure 3. Architecture of Neurocognitive Empowerment for Addiction Treatment (NEAT) for 14 sessions. In each session a new cognitive domain is added to the previous one in trainings and exercises (accumulative architecture). The brightness of color indicates the level of complexity of the cognitive modules. The basic modules (e.g., sustained attention) marked with bright colors and the complex ones marked with dark colors (e.g., monitoring)

\subsection{Data management}

Confidentiality will be maintained by keeping identifying information (such as names and phone numbers) in secure servers. Except for a contact form, research data will be identified by a subject number rather than by name. An alpha numeric system will be used for identification of subjects. All hard copies of study information will be kept in locked file cabinets inside a locked office. All electronic study information will be stored on secure servers with appropriate access controls. Only study personnel will have access to the identifying data. WIR staff will be aware of who is participating in the research study, as they will know who is attending this group versus the other WIR activities planned at that time. In addition, these treatment providers may insert into their treatment records that they attended these treatment sessions. 
However, treatment program staff will not be privy to any of the information provided to research study personnel via interview, behavioral, or self-report assessments. Subject identity will not be disclosed to any person who is not authorized to see this information, except in the event of a medical emergency or if required by law. Paper copies of consents, screening forms, the Research Privacy Form and any other forms, and testing results or papers containing Personally Identifiable Information (PII) will be stored in locked cabinets at the Laureate Institute for Brain Research (LIBR). Any electronic data will have all identifiable information encrypted and be stored on a password-protected database on a secure server managed by LIBR.

Participants will be told that their information provided during the course of the study will remain confidential within the research study team. However, given the nature of the treatment groups held at WIR, confidentiality concerning their participation in the study cannot be guaranteed. Family members will be allowed to be present and discuss the consenting process with the participant if requested.

\begin{tabular}{|c|c|c|c|c|c|c|c|c|c|c|c|c|c|}
\hline & Screen & Pre- & $\begin{array}{c}\text { Week } \\
1\end{array}$ & $\begin{array}{c}\text { Week } \\
2\end{array}$ & $\begin{array}{c}\text { Week } \\
\mathbf{3}\end{array}$ & $\begin{array}{c}\text { Week } \\
4\end{array}$ & $\begin{array}{c}\text { Week } \\
5\end{array}$ & $\begin{array}{c}\text { Week } \\
6\end{array}$ & $\begin{array}{c}\text { Week } \\
7\end{array}$ & Post- & $\begin{array}{l}3 \text { Month } \\
\text { Follow Up }\end{array}$ & $\begin{array}{l}6 \text { Month } \\
\text { Follow Up }\end{array}$ & $\begin{array}{l}12 \text { Month } \\
\text { Follow Up }\end{array}$ \\
\hline \multicolumn{14}{|l|}{ Enrollment } \\
\hline Eligibility Screen & + & & & & & & & & & & & & \\
\hline Informed Consent & + & & & & & & & & & & & & \\
\hline Allocation & & + & & & & & & & & & & & \\
\hline \multicolumn{14}{|l|}{ Intervention } \\
\hline NEAT Intervention & & & $\leftarrow$ & & & & & & $\longrightarrow$ & & & & \\
\hline Active Control & & & $\longleftarrow$ & & & & & & $\longrightarrow$ & & & & \\
\hline \multicolumn{14}{|l|}{ Assessments } \\
\hline $\begin{array}{c}\text { OCDUS, ASI, CDDR, DDQ, TEQ } \\
\text { BIS/BAS, UPPS-P, DERS, PROMIS } \\
\text { Scales (for substance use and } \\
\text { mental health) }\end{array}$ & & + & & & & & & & & + & + & + & + \\
\hline SES, CEST & & & + & + & + & + & + & + & + & & + & + & + \\
\hline HRS,WAI, Subjective Feedback & & & + & + & + & + & + & + & + & & & & \\
\hline All Other Self Report Measures & & + & & & & & & & & + & + & + & + \\
\hline Neuropsychological Assessments & & + & & & & & & & & + & & & \\
\hline Neuroimaging Assessments & & + & & & & & & & & + & & & \\
\hline Urine Drug Tests & & + & + & + & + & + & + & + & + & + & + & + & + \\
\hline
\end{tabular}

Figure 4. Schedule of enrolment, interventions and assessments. This figure indicates the assessments or interventions completed for screening, pre-intervention, weekly during completion of intervention, post intervention, and 3- and 6month follow-up. Participants are randomized in groups of 9-12 to complete either neuroscience-informed psychoeducation (NEAT) or active control intervention. OCDUS: Obsessive Compulsive Drug Use Scale, ASI: Addiction Severity Index, CDDR: Customary Drinking and Drug Use Record, DDQ: Desire for Drug Questionnaire, TEQ: Traumatic Events Questionnaire, BIS/BAS: Behavioral Inhibition System/Behavioral Approach Scale, UPPS-P: UPPS Impulsive Behavior Scale, DERS: Difficulties in Emotion Regulation Scale, PROMIS: Patient-Reported Outcomes Measurement Information System, SES: Services Engagement Scale, CEST: Client Evaluation of Self and Treatment, HRS: Homework Rating Scale, WAI: Working Alliance Inventory, PROMIS-CA: PROMIS Cog Abilities, PROMIS-CG: PROMIS Cog General

\subsection{Statistical analysis}

Categorical variables will be summarized by frequency and proportion, and continuous variables by mean and standard deviation (SD), or by median and inter-quartile range if the distribution appeared skewed even after simple (e.g. log) transformations, for all participants and for participants in each treatment arm. Data normality will be evaluated by quantile-quantile (QQ) plot and Shapiro-Wilk test.

Treatment effects will be compared by the intention-to-treat (ITT) principle, i.e., all subjects who were randomized will be included in the final analysis, regardless of their adherence to any of the interventions. In order to detect changes in the variables recorded for the primary and secondary outcomes, mixedeffects model analyses will be performed using fixed-effects of time (pre-, post-intervention time points) as a within subjects factor and condition (intervention vs. control) as a between-subjects factor; withinsubject dependency will be captured by either random intercepts or within-subject correlation structure (e.g. $1^{\text {st }}$-order autoregressive or exponential power), whichever Akaike or Bayesian information criterion (AIC or BIC) favor. We include age and IQ as covariates. The inclusion of other covariates will be 
determined by AIC and BIC. Subgroup analyses will be conducted according to the baseline neuropsychological and fMRI characteristics. Significance level will be set at 0.05 level for the primary outcome and hypothesis; Bonferroni correction will be applied to all pre-post comparisons for all secondary outcomes, and Benjamini-Hochberg's procedure to control false discovery rate at $5 \%$ level for all the exploratory analyses. For each outcome measure (primary, secondary, and exploratory), effect sizes of Cohen's $d$ and $f$ will be computed. The effect sizes, along with attrition rate at each time point, can be used to guide future study design.

Table 1 Diagnostic, demographic, self-report, behavioral, and neuroimaging assessments

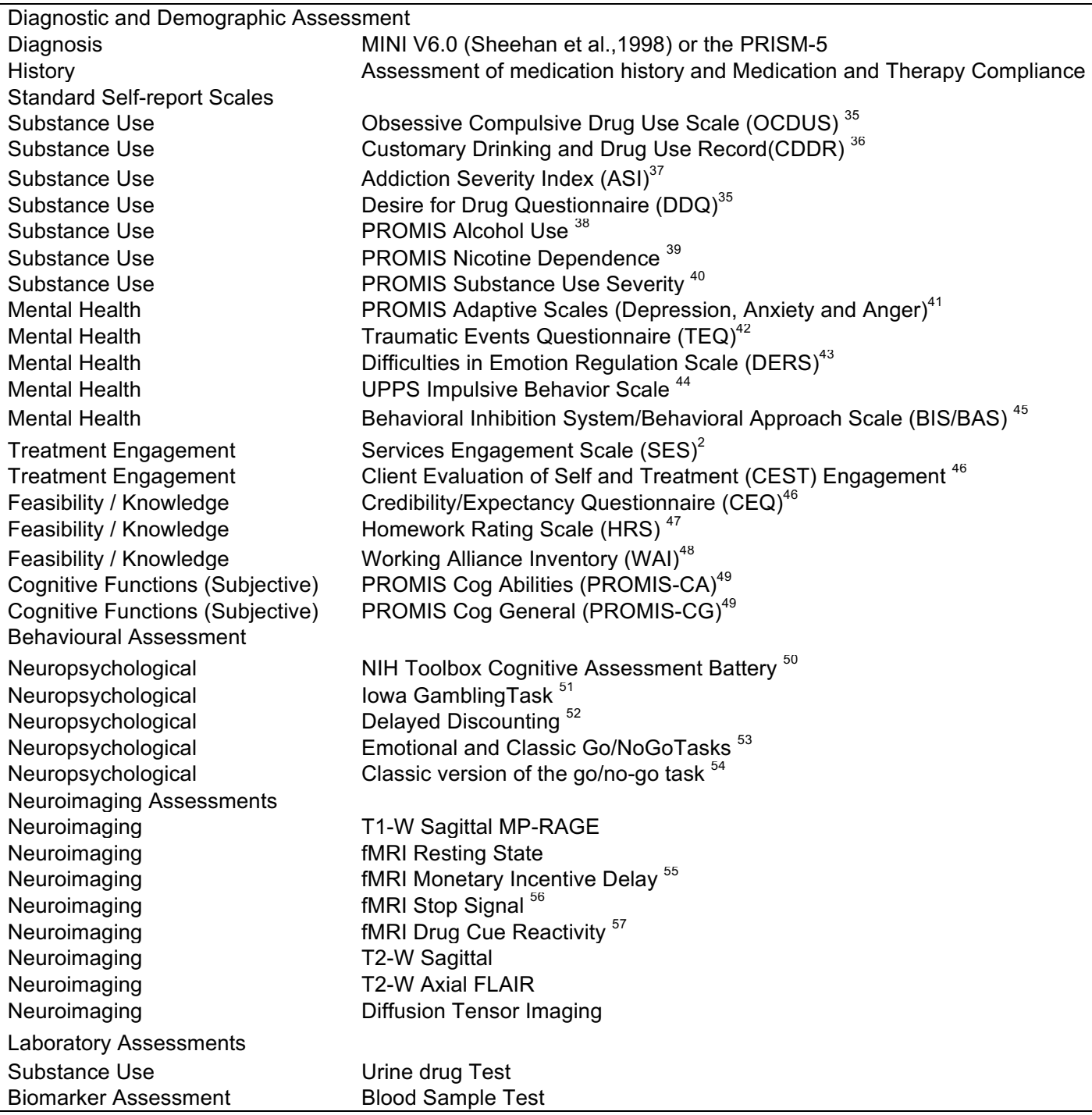

\section{Discussion}

In many people with substance use disorders, lack of self-awareness on how their brains behave to develop craving and initiate relapse is a complication that hampers efforts for abstinence and delays progress in recovery ${ }^{51,58}$. Given this deficit, this group of patients are well-equipped to manage their craving and may deny drug-related problems and underestimate the need for treatment ${ }^{59,60}$. Enhancing 
self-awareness by training neurocognitive skills to help patients to deal with drug craving and other cognitive consequences of chronic drug use is the aim of this study that was described in detail above. In this work, we developed a new neurocognitive rehabilitation program (NEAT) that addresses neurocognitive deficits reported by previous studies in substance users ${ }^{12,61,62}$. The unique aspect of NEAT is using interactive and engaging structure (including cartoons, brain awareness games and real-life scenarios) to train the complex content of neurocognitive functions (e.g., interoception, episodic future thinking, inhibition), metacognitive and compensatory skills (e.g., self-monitoring, goal management training).

We assume that compared to the control intervention, the patients who receive NEAT program, indicate positive changes in their roadmap for recovery during the course of study. These changes are measured by using clinical, psychological, and neuropsychological assessments as well as neuroimaging techniques. Fewer studies before have indicated the effectiveness of metacogitive awareness and cognitive rehabilitation interventions in alcohol and substance users, in terms of craving control, improved cognitive functions and better treatment outcomes (e.g., treatment adherence, length of abstinence) ${ }^{63-69}$. Although in all these studies, the promising results are viewed as a sign of improved regulatory control of top-down system over bottom-up system, but no evidence exists for underlying neural substrates. Therefore, our study is the first attempt that apply a broad range of assessments including neural components, to monitor the effect of cognitive rehabilitation program in substance users. We also consider the effect of intervention on real world functioning (e.g., craving management and social and emotional competency) to provide more data on the ecological validity. Randomized allocation of patients in two groups, employment of an active control condition, blinding outcome assessors, using a fully manualized intervention, monitoring the long-term effect of intervention during one-year follow-up are the most important strengths of the present study.

In this study we are expecting to face following limitations: (1) participant withdrawal due to schedules changing or phasing up in the program, (2) scheduling of other appointments (i.e. visiting a physician) during Brain Gym times, (3) participants not completing suggested homework or not fill the brain planner, (4) participants absconding from WiR program or being terminated from program, (5) follow up problem and lack of data about those participants once they are discharged from WiR.

To conclude, the effects of a multi-component neurocognitive rehabilitation and awareness program termed 'Neurocognitive Empowerment for Addiction Treatment' (NEAT) on psychological, neurocognitive and structural and functional neuroimaging measures will be examined in a group of female opioids and or methamphetamine users in the context of addiction recovery. If the results of this study prove the efficacy of NEAT as it is expected, then it could be transferred to the clinical practice and embedded into the routine therapeutic programs as a supplementary intervention.

\section{Trial status}

The present trial is ongoing. Recruitment commenced in January 2019. Data collection is predicted to be completed by the end of 2021.

\begin{tabular}{ll}
\multicolumn{2}{l}{ List of abbreviations } \\
ASI & Addiction Severity Index \\
BIS/BAS & Behavioral Inhibition System/Behavioral Approach Scale \\
CEST & Client Evaluation of Self and Treatment \\
CEQ & Credibility/Expectancy Questionnaire \\
CDDR & Customary Drinking and Drug Use Record \\
DCM & Dynamic model of craving \\
DDQ & Desire for Drug Questionnaire
\end{tabular}




$\begin{array}{ll}\text { DERS } & \text { Difficulties in Emotion Regulation Scale } \\ \text { HRS } & \text { Homework Rating Scale } \\ \text { MINI } & \text { Mini-International Neuropsychiatric Interview } \\ \text { MUD } & \text { Methamphetamine use disorder } \\ \text { NCDs } & \text { Neurocognitive deficits } \\ \text { NEAT } & \text { Neurocognitive Empowerment for Addiction Treatment } \\ \text { OCDUS } & \text { Obsessive Compulsive Drug Use Scale } \\ \text { OUD } & \text { Opioid use disorder } \\ \text { RCT } & \text { Randomized controlled trial } \\ \text { SES } & \text { Services Engagement Scale } \\ \text { TAU } & \text { Treatment-as-usual } \\ \text { TEQ } & \text { Traumatic Events Questionnaire } \\ \text { WAI } & \text { Working Alliance Inventory } \\ \text { WIR } & \text { Women in Recovery }\end{array}$

\section{Declarations \\ Acknowledgements}

Authors would like to thank WiR staff who have been seminal for this study to be designed and started. Authors also thank Tim Collins and Katie Redman who have contributed to put all pieces of assessments together at LIBR. The role of Naeim Tadayon (NEAT workbook illustrator) and Mohsen Farhadi (NEAT workbook graphic designer) is also highly appreciated to make the intervention package ready to go.

\section{Funding}

This study is funded by Oklahoma Center for Advancement of Science and Technologies (OCAST) (grant number HR18-139) from July 1st, 2019 (after one-year extension no cost) for 3 years. OCAST has no role in study design; collection, management, analysis, and interpretation of data; writing of the report; and the decision to submit the report for publication.

\section{Availability of data and materials}

The data that will be collected in this study will be available from the principal investigator on reasonable request.

\section{Dissemination policy}

When the data collection is completed, the total data set will be analyzed, and the results will be published in scientific journals and presented at (inter)national scientific meetings. A summary of the results will be released to the participants of this study. The identity of the participants will not be disclosed in any of these publication forms. The researchers of this study will attempt to reduce the time between the completion of data collection and the release of the study results.

\section{Authorship}

The principal investigators will justify the names for authorship. Individuals who fulfil authorship criteria will be an author on the manuscripts.

\section{Authors' contributions}

$H E$, RA, and MP designed the study. HE and TR developed the initial draft of the manuscript. HY and RK developed the statistical and imaging part of the methods. HY also generated randomization codes. All authors read, revised and approved the final draft of the manuscript. 


\section{Ethics approval and consent to participate}

The trial was approved by Western Institutional Review Board as an Independent IRB in WIRB-Copernicus Group Company 1019 39th Avenue SE Suite 120, Puyallup, WA 98374-2115. NEAT unique study ID in LIBR is 2018-007 with IRB approval number: 20181028, 1-1141403-1. The purpose and potential risks of this clinical trial will be fully explained to the participants and their families. All participants will be asked to provide written informed consent before participation.

\section{Pre-registration}

This study is registered in clinicaltrials.gov on 22 April 2019 with NCT03922646 as clinicalTrials.gov identifier.

\section{Competing interests}

$\mathrm{HE}, \mathrm{TR}, \mathrm{MP}$ and RA were involved with the NEAT program development; however, they were all blinded to the assessment procedures or data analysis.

Consent for publication

Not applicable.

\section{References}

American Psychiatric Association. Diagnostic and statistical manual of mental disorders. 4th ed. Washington, DC: APA; 1994.

Amorim, P.(2000). Mini International Neuropsychiatric Interview (MINI): validation of a short structured diagnostic psychiatric interview. Rev Bras Psiquiatr. 22(3):106-15.

1. Crist RC, Clarke TK, Berrettini WH. Pharmacogenetics of Opioid Use Disorder Treatment. CNS Drugs. 2018;32(4):305-320.

2. Tait RJ, Whetton S, Shanahan M, et al. Quantifying the societal cost of methamphetamine use to Australia. Int J Drug Policy. 2018;62:30-36.

3. Jones MR, Viswanath O, Peck J, Kaye AD, Gill JS, Simopoulos TT. A Brief History of the Opioid Epidemic and Strategies for Pain Medicine. Pain Ther. 2018;7(1):13-21.

4. Chan B, Freeman $M$, Kondo $\mathrm{K}$, et al. Pharmacotherapy for methamphetamine/amphetamine use disorder-a systematic review and meta-analysis. Addiction (Abingdon, England). 2019;114(12):2122-2136.

5. Hedegaard H BB, Trinidad JP, Spencer M, Warner M. Drugs Most Frequently Involved in Drug Overdose Deaths: United States, 2011-2016. National Vital Statistics Reports. 2018;67(9):1-12.

6. Martins SS, Fenton MC, Keyes KM, Blanco C, Zhu H, Storr CL. Mood and anxiety disorders and their association with non-medical prescription opioid use and prescription opioiduse disorder: longitudinal evidence from the National Epidemiologic Study on Alcohol and Related Conditions. Psychol Med. 2012;42(6):1261-1272.

7. Watt MH, Myers, B., Towe, S. L., \& Meade, C. S. . The mental health experiences and needs of methamphetamine users in Cape Town: A mixed methods study. South African medical journal = Suid-Afrikaanse tydskrif vir geneeskunde. 2015;105(8):685-688.

8. Rezapour T, DeVito EE, Sofuoglu M, Ekhtiari H. Perspectives on neurocognitive rehabilitation as an adjunct treatment for addictive disorders: From cognitive improvement to relapse prevention. Progress in brain research. 2016;224:345-369.

9. Bruijnen C, Dijkstra BAG, Walvoort SJW, et al. Prevalence of cognitive impairment in patients with substance use disorder. Drug Alcohol Rev. 2019;38(4):435-442. 
10. Ersche KD, Sahakian BJ. The neuropsychology of amphetamine and opiate dependence: implications for treatment. Neuropsychol Rev. 2007;17(3):317-336.

11. leong HF, Yuan Z. Resting-State Neuroimaging and Neuropsychological Findings in Opioid Use Disorder during Abstinence: A Review. Front Hum Neurosci. 2017;11:169.

12. Cadet JL, Bisagno V. Neuropsychological Consequences of Chronic Drug Use: Relevance to Treatment Approaches. Front Psychiatry. 2015;6:189.

13. Volkow ND, Fowler JS, Wang G-J. The addicted human brain: insights from imaging studies. Journal of Clinical Investigation. 2003;111(10):1444-1451.

14. Sofuoglu M, DeVito EE, Waters AJ, Carroll KM. Cognitive Function as a Transdiagnostic Treatment Target in Stimulant Use Disorders. J Dual Diagn. 2016;12(1):90-106.

15. Bates ME VG, Buckman JF, Labouvie EW, Barry D. Short term neuropsychological recovery in substance use disordered clients. Alcohol Clin Exp Res. 2005;29(3):367-377.

16. Sofuoglu M, DeVito EE, Waters AJ, Carroll KM. Cognitive enhancement as a treatment for drug addictions. Neuropharmacology. 2013;64:452-463.

17. Cicerone KD, Langenbahn DM, Braden C, et al. Evidence-based cognitive rehabilitation: updated review of the literature from 2003 through 2008. Arch Phys Med Rehabil. 2011;92(4):519-530.

18. Cicerone KD, Goldin Y, Ganci K, et al. Evidence-Based Cognitive Rehabilitation: Systematic Review of the Literature From 2009 Through 2014. Arch Phys Med Rehabil. 2019;100(8):1515-1533.

19. O'Brien AR, Chiaravalloti N, Goverover Y, Deluca J. Evidenced-based cognitive rehabilitation for persons with multiple sclerosis: a review of the literature. Arch Phys Med Rehabil. 2008;89(4):761-769.

20. Barlati S, Deste G, De Peri L, Ariu C, Vita A. Cognitive remediation in schizophrenia: current status and future perspectives. Schizophr Res Treatment. 2013;2013:156084.

21. Sheehan D, Lecrubier, Y., Sheehan, H., et al. The Mini International Neuropsychiatric Interview (MINI). A short diagnostic structured interview: reliability and validity according to the CIDI. J Clin Psychiatry. 1997;12(5):224-231.

22. Rezapour T, Aupperle RL, Paulus MP, Ekhtiari H. Clinical translation and implementation neuroscience for novel cognitive interventions in addiction medicine. Cognition and Addiction2020:393-404.

23. Beck AT. The past and future of cognitive therapy. J Psychother Pract Res. 1997;6(4):276284.

24. Rezapour T, Hatami, J, Farhoudian, A, Sofuoglu, M, Noroozi, A, Daneshmand, R, Samiei, A, Ekhtiari, H. NEuro COgnitive REhabilitation for Disease of Addiction (NECOREDA) Program: From Development to Trial. Basic Clin Neurosci. 2015;6(4):291-298.

25. Ekhtiari H, Rezapour T, Aupperle RL, Paulus MP. Neuroscience-informed psychoeducation for addiction medicine: A neurocognitive perspective. Progress in brain research. 2017;235:239-264.

26. Rezapour T, Hatami J, Farhoudian A, et al. Cognitive rehabilitation for individuals with opioid use disorder: A randomized controlled trial(). Neuropsychol Rehabil. 2019;29(8):1273-1289.

27. Maraver MJ, Bajo MT, Gomez-Ariza CJ. Training on Working Memory and Inhibitory Control in Young Adults. Front Hum Neurosci. 2016;10:588. 
28. Fukuba E, Kitagak,i H., Wada, A., Uchida, K., Hara, S., Hayashi, T., Oda, K., Uchida,N. Brain Activation during the Spot the Differences Game. Magnetic Resonance in Medical Sciences 2009;8(1):23-32.

29. López-Luengo B, Vázquez C. Effects of Attention Process Training on cognitive functioning of schizophrenic patients. Psychiatry Research. 2003;119(1-2):41-53.

30. Dymowski AR, Ponsford JL, Willmott C. Cognitive training approaches to remediate attention and executive dysfunction after traumatic brain injury: A single-case series. Neuropsychol Rehabil. 2016;26(5-6):866-894.

31. Copersino ML. Cognitive Mechanisms and Therapeutic Targets of Addiction. Curr Opin Behav Sci. 2017;13:91-98.

32. Barman A, Chatterjee, A., \& Bhide, R. Cognitive Impairment and Rehabilitation Strategies After Traumatic Brain Injury. Indian journal of psychological medicine. Indian journal of psychological medicine. 2016;38(3):172-181.

33. Huckans M, Hutson L, Twamley E, Jak A, Kaye J, Storzbach D. Efficacy of cognitive rehabilitation therapies for mild cognitive impairment $(\mathrm{MCl})$ in older adults: working toward a theoretical model and evidence-based interventions. Neuropsychol Rev. 2013;23(1):63-80.

34. Plaete J, De Bourdeaudhuij I, Verloigne M, Crombez G. The use and evaluation of selfregulation techniques can predict health goal attainment in adults: an explorative study. PeerJ. 2016;4:e1666.

35. Frankena I, Hendriksa, V, Brink. W. Initial validation of two opiate craving questionnaires The Obsessive Compulsive Drug Use Scale and the Desires for Drug Questionnaire. Addictive Behaviors. 2002;27(5):675-685.

36. Brown S, Myers, MG, Lippke, L, Tapert, SF, Stewart, DG, Vik, PW. Psychometric evaluation of the customary drinking and drug use record (CDDR): A measure of adolescent alcohol and drug involvement. Journal of studies on alcohol and drugs. 1998;59(4):427-438.

37. Kosten T. Concurrent validity of the Addiction Severity Index. The journal of nervous and mental disease. 1983;171(10):606-610.

38. Pilkonis PA, Yu L, Colditz J, et al. Item banks for alcohol use from the Patient-Reported Outcomes Measurement Information System (PROMIS): use, consequences, and expectancies. Drug Alcohol Depend. 2013;130(1-3):167-177.

39. Shadel WG, Edelen MO, Tucker JS, Stucky BD, Hansen M, Cai L. Development of the PROMIS nicotine dependence item banks. Nicotine Tob Res. 2014;16 Suppl 3:S190-201.

40. Pilkonis PA, Yu L, Dodds NE, et al. Item banks for substance use from the Patient-Reported Outcomes Measurement Information System (PROMIS((R))): Severity of use and positive appeal of use. Drug Alcohol Depend. 2015;156:184-192.

41. Pilkonis PA, Choi SW, Reise SP, et al. Item banks for measuring emotional distress from the Patient-Reported Outcomes Measurement Information System (PROMIS(R)): depression, anxiety, and anger. Assessment. 2011;18(3):263-283.

42. Vrana S, Lauterbach, D. Prevalence of Traumatic Events and Post-Traumatic Psychological Symptoms in a Nonclinical Sample of College Students. Journal of Traumatic Stress. 1994;7:289-302.

43. Gratz KL, Roemer, L. . Multidimensional Assessment of Emotion Regulation and Dysregulation: Development, Factor Structure, and Initial Validation of the Difficulties in 
Emotion Regulation Scale. Journal of Psychopathology and Behavioral Assessment 2004;26:41-54.

44. Whiteside SP, Lynam DR, Miller JD, Reynolds SK. Validation of the UPPS impulsive behaviour scale: a four-factor model of impulsivity. European Journal of Personality. 2005;19(7):559-574.

45. Carver CS, White TL. Behavioral inhibition, behavioral activation, and affective responses to impending reward and punishment: The BIS/BAS Scales. Journal of Personality and Social Psychology. 1994;67(2):319-333.

46. Devillya GJ, Borkovec, T.D. Psychometric properties of the Credibility/Expectancy Questionnaire. Journal of Behavior Therapy and Experimental Psychiatry. 2000;31(2):7386.

47. Kazantzis N, Deane FP, Ronan KR. Assessing compliance with homework assignments: review and recommendations for clinical practice. J Clin Psychol. 2004;60(6):627-641.

48. Hatcher RL, Gillaspy JA. Development and validation of a revised short version of the working alliance inventory. Psychotherapy Research. 2006;16(1):12-25.

49. Becker $\mathrm{H}$, Stuifbergen A, Lee $\mathrm{H}$, Kullberg V. Reliability and Validity of PROMIS Cognitive Abilities and Cognitive Concerns Scales Among People with Multiple Sclerosis. Int J MS Care. 2014;16(1):1-8.

50. Tulsky DS, Carlozzi NE, Chevalier N, Espy KA, Beaumont JL, Mungas D. V. NIH Toolbox Cognition Battery (CB): measuring working memory. Monogr Soc Res Child Dev. 2013;78(4):70-87.

51. Goldstein RZ, Craig AD, Bechara A, et al. The neurocircuitry of impaired insight in drug addiction. Trends Cogn Sci. 2009;13(9):372-380.

52. Odum AL. Delay discounting: I'm a k, you're a k. J Exp Anal Behav. 2011;96(3):427-439.

53. Cohen-Gilbert JE, Thomas KM. Inhibitory control during emotional distraction across adolescence and early adulthood. Child Dev. 2013;84(6):1954-1966.

54. Guitart-Masip M, Economides M, Huys QJ, et al. Differential, but not opponent, effects of L -DOPA and citalopram on action learning with reward and punishment. Psychopharmacology (Berl). 2014;231(5):955-966.

55. Knutson B, Bhanji, J. P., Cooney, R. E., Atlas, L. Y., \& Gotlib, I. H. Neural responses to monetary incentives in major depression. Biological psychiatry. 2008;63(7):686-692.

56. Matthews S, Simmons, AN., Arce, E., Paulus, MP. Dissociation of inhibition from error processing using a parametric inhibitory task during functional magnetic resonance imaging. Neuroreport. 2005;16(7):755-760.

57. Ekhtiari H, Kuplicki R, Pruthi A, Paulus M. Methamphetamine and Opioid Cue Database (MOCD): Development and Validation. Drug Alcohol Depend. 2020;209:107941.

58. Castine BR, Albein-Urios N, Lozano-Rojas O, Martinez-Gonzalez JM, Hohwy J, VerdejoGarcia A. Self-awareness deficits associated with lower treatment motivation in cocaine addiction. Am J Drug Alcohol Abuse. 2019;45(1):108-114.

59. Dean AC, Kohno M, Morales AM, Ghahremani DG, London ED. Denial in methamphetamine users: Associations with cognition and functional connectivity in brain. Drug Alcohol Depend. 2015;151:84-91. 
60. Ferrari JR, Groh DR, Rulka G, Jason LA, Davis MI. Coming to Terms With Reality: Predictors of Self-deception Within Substance Abuse Recovery. Addict Disord Their Treat. 2008;7(4):210-218.

61. J. GT. Addiction and cognition. Addiction science \& clinical practice. 2010;5(2):4-14.

62. Kiluk BD, Nich C, Carroll KM. Relationship of cognitive function and the acquisition of coping skills in computer assisted treatment for substance use disorders. Drug Alcohol Depend. 2011;114(2-3):169-176.

63. Bell MD, Laws HB, Petrakis IB. A randomized controlled trial of cognitive remediation and work therapy in the early phase of substance use disorder recovery for older veterans: Neurocognitive and substance use outcomes. Psychiatr Rehabil J. 2017;40(1):94-102.

64. Bickel WK, Yi R, Landes RD, Hill PF, Baxter C. Remember the future: working memory training decreases delay discounting among stimulant addicts. Biol Psychiatry. 2011;69(3):260-265.

65. Gamito P, Oliveira J, Lopes $\mathrm{P}$, et al. Executive functioning in alcoholics following an mHealth cognitive stimulation program: randomized controlled trial. J Med Internet Res. 2014;16(4):e102.

66. Marceau EM, Berry J, Lunn J, Kelly PJ, Solowij N. Cognitive remediation improves executive functions, self-regulation and quality of life in residents of a substance use disorder therapeutic community. Drug Alcohol Depend. 2017;178:150-158.

67. Rass O, Schacht RL, Buckheit K, Johnson MW, Strain EC, Mintzer MZ. A randomized controlled trial of the effects of working memory training in methadone maintenance patients. Drug Alcohol Depend. 2015;156:38-46.

68. Wanmaker S, Leijdesdorff SMJ, Geraerts E, van de Wetering BJM, Renkema PJ, Franken IHA. The efficacy of a working memory training in substance use patients: A randomized double-blind placebo-controlled clinical trial. J Clin Exp Neuropsychol. 2018;40(5):473486.

69. Zhu Y, Jiang, H., Su, H., Zhong, N., Li, R., Li, X., Chen, T., Tan, H., Du, J., Xu, D., Yan, H., Xu, D., \& Zhao, M. . A Newly designed mobile-based Computerized Cognitive Addiction Therapy (CCAT) App improved cognition impairments and risk decision making inmethamphetamine use disorder: A randomized clinical study. JMIR mHealth and uHealth. 2018;6(6):1-27. 